Please do not remove this page

RMIT

UNIVERSITY

\title{
Development of a conductive photoresist with a mixture of SU-8 and HCL doped polyaniline
}

Annaiyan, Uma; Kalantar Zadeh, Kourosh; Fang, Qiang; Cosic, Irena

https://researchrepository.rmit.edu.au/esploro/outputs/9921859595101341/filesAndLinks?institution=61RMIT_INST\&index=null

Annaiyan, U., Kalantar Zadeh, K., Fang, Q., \& Cosic, I. (2005). Development of a conductive photoresist with a mixture of SU-8 and HCL doped polyaniline. Proceedings of the IEEE TENCON Region 10

Conference, 1-5. https://doi.org/10.1109/TENCON.2005.300927

Published Version: https://doi.org/10.1109/TENCON.2005.300927

Repository homepage: https://researchrepository.rmit.edu.au

(c) 2005 IEEE. Personal use of this material is permitted. However, permission to reprint/republish this material for advertising or promotional purposes or for creating new collective works for resale or redistribution to servers or lists, or to reuse any copyrighted component of this work in other works must be obtained from the IEEE.

Downloaded On 2023/04/26 22:50:34 +1000 


\title{
Development of a conductive photoresist with a mixture of SU-8 and $\mathrm{HCl}$ doped polyaniline
}

\author{
Uma M Annaiyan*, Kourosh Kalantar-Zadeh, Qiang Fang, Irena Cosic
}

\begin{abstract}
A novel electrically conductive photoresist has been formulated to fabricate microcomponents. The developed conductive photoresist is based upon SU-8 photopolymer, an insulating negative-tone epoxy, in which protonically doped polyaniline (PANI) nanoparticles have been dispersed to enhance the electrical properties. The characteristics of this new conductive photoresist have been studied via electrical measurements. The process for preparing the conductive films from the combination of EB (Emeraldine base) PANI, SU-8 and NMP (N-methyl-2-pyrrolidinone) will be presented. Different weight percentages (wt \%) of SU-8 in the above combination have been prepared and spin coated to form thin-films which have been protonated with $\mathrm{HCl}$. The conductivities of the thinfilms were measured using a four point probe. The highest conductivity achieved was approximately $1.6 \mathrm{~S} / \mathrm{cm}$ for the mixture of $10 \mathrm{wt} \%$ of SU-8 25 with EB-PANI mixed with NMP. The morphology of the thin-films was studied using a Scanning Electron Microscopy (SEM).
\end{abstract}

Index Terms - SU-8, photoresist, polyaniline, conductive polymer, protonation, biosensors.

\section{INTRODUCTION}

$\mathrm{C}$ urrent microfabrication processes are used to develop $C_{\text {devices with dimensions in the sub-micrometer to }}$ millimeter range. Such processes the development of devices such as micro-sensors and micro-actuators. Micromachining techniques for fabricating three-dimensional structures differ from conventional, sequential machining processes, such as drilling or milling with mechanical tools [1]. Integrated circuit fabrication processes are the most prevalent example of microfabrication [2-4]. Microfabrication also presents the

This work was supported in part by the Royal Melbourne University of Technology, Australia under RIF Grant.

Uma M. Annaiyan, is with the School of Electrical and Computer Engineering Department, Royal Melbourne Institute of Technology, Melbourne, VIC 3001, AUSTRALIA. Royal Melbourne Institute of Technology, Melbourne, VIC 3001, AUSTRALIA (Corresponding author : phone: 61-399259533; fax: 61-3-99252007; email: s2118358@student.rmit.edu.au).

* Kourosh Kalantar-Zadeh, Lecturer, is with the Royal Melbourne Institute of Technology, Melbourne, VIC 3001, AUSTRALIA (e-mail: kourosh.kalantar@rmit.edu.au).

Qiang Fang, Lecturer is with the School of Electrical and Computer Engineering Department, Royal Melbourne Institute of Technology, Melbourne, VIC 3001, AUSTRALIA (e-mail: john.fang@rmit.edu.au).

Irena Cosic, Professor and Head of School, is with the School of Electrical and Computer Engineering Department, Royal Melbourne Institute of Technology, Melbourne, VIC 3001, AUSTRALIA (e-mail: irena.cosic@rmit.edu.au). possibility of batch processing, the optional integration of electronics and the reproducibility of sensor characteristics due to the accurate geometric control in the fabrication process [1]. The four basic microfabrication techniques are: deposition, patterning, doping and etching. These techniques are combined to build up a device layer by layer. Generally thin- films are deposited on top of the substrate as active or passive components. Microfabrication lithography techniques rely on radiation-sensitive polymers called resists. The resist is irradiated through a mask containing the pattern to be transferred, resulting in cross-linking, which creates a difference in solubility between the irradiated and unexposed areas of the polymer [5]. Finally, the pattern is transferred from the photoresist layer to the substrate by etching or lift off techniques. After removing the remaining photoresist, the next layer is deposited and structured. Doping of a semiconductor material can be undertaken directly after photolithography or after patterning an implantation mask. Doping techniques are typically undertaken to modify the electrical conductivity of materials which are patterned. Hence it is a key step in the fabrication of semiconductor devices such as diodes and transistors.

Each of the previously mentioned steps, thin-film deposition, photolithography and doping, increases the cost of fabrication. Having a photoresist with a controlled conductivity will dramatically reduce the fabrication process time as the photoresist layer can be directly implemented as the semiconducting material. Utilisation of a conductive photoresist eliminates the etching process and simplifies the microfabrication process. Additionally, such a material can also be of tremendous benefit in the fields of sensors and actuators where chemical, electrical and mechanical properties are the key parameters in the development of micro-devices. There are other advantages that this material can offer. For instance for the fabrication of high aspect ratio devices such as spikes which have many applications for biosensing measurements. The silicon etching process currently used to develop such structures has inherent deficiencies such as: slow etch rate, difficulties in applying external connections, lack of control in the conductivity of structures and incompatibility with layered device fabrication. All these problems could be solved with a conductive photoresist with controllable conductivity. 
In order to develop a conductive photoresist a novel approach of mixing a conductive polymer with the photoresist is presented in this paper. SU-8 is used as the base photoresist. To improve the functionality of the SU-8, it was doped with a conductive polymer. The goal is to load the SU8 epoxy with conductive particles in order to enhance its electrical conductivity. In this paper, we use polyaniline (PANI), a conductive polymer. A different approach to develop conductive SU-8 was initiated by Jiguer et al [6] where they combined silver nano-composites with SU-8.

SU-8 is a negative tone, chemically amplified, near UV photoresist which was first developed at IBM Yorktown, USA in the late 1980s [7]. The specific properties of SU-8 enable ultra-thick layers of high uniformity with low edge bead, high transparency at wavelengths above $340 \mathrm{~nm}$, low optical absorption [8] and smooth surface up to $1 \mathrm{~mm}$ by a single coating step. Near UV exposure with low doses yields patterns with nearly vertical sidewalls of high aspect ratio $>50$ [9-11]. Ultra thick resists with high aspect ratios are essential for several MEMS, bioMEMS and MOEMS applications, such as electrostatic sensors and actuators, fluidic channels, nanobiosensors and micro robots [12], microelectronics, micromechanics, microfluidics, packaging, magnetics, microoptics, at panel displays, and microwave devices [5]. SU-8 is also sensitive to electron beam exposure and has several advantages in applications when compared to the most widely used e-beam resist, polymethyl methacrylate (PMMA) [13]. Firstly SU-8 has a much higher sensitivity which greatly reduces the time required to expose a given pattern. Secondly, cross linked (fully exposed) SU-8 is an optically transparent epoxy that is chemically and physically durable. For many applications these properties eliminate the need for a dry-etch pattern transfer onto a more durable substrate [13]. The SU-8 material consists of an epoxy called EPON SU-8, a solvent called gamma-butyrolactone (GBL) and a photoinitiator taken from the family of the triaryliumsulfonium salts [14].

It is possible to create conductive polymers with a diverse range of properties [15]. The material class of conducting polymers is used in actuators, transistors, wires, super capacitors, strain gauges [15], electromagnetic interference (EMI) shielding, electrostatic discharge (ESD) protection, metallization, lithography-(e.g., charge dissipaters, conducting resists), corrosion protection of metals [5], fibers, antistatic and other coating, films, diodes, electroluminescent devices, displays, printed circuit boards, electrochromic windows in houses and cars, conductive fabrics [16], optoelectronic devices [17] and much more. The use of a polymer as a component material for the biosensing applications provides the sensors with very high sensitivity and convenient mechanical properties. Conductive polymers such as PANI are increasingly applied in biosensor construction for the sensing process [5].

Polyaniline (PANI) is one of the most popular and chemically stable polymers [18] under ambient conditions, having high conductivity [19]. PANI has been described in many papers for the past 120 years [20-23]. It is most promising because of its straight forward polymerization $[24,25]$. The conductivity of PANI depends on various factors such as oxidizing agents, aniline/oxidant mole ratio $(\mathrm{k})$, reaction temperature and time of polymerization [26], the percentage of protonation and the degree of water and solvent content [27]. The term 'PANI' is widely used to indicate any polymeric material obtained by the oxidation of aniline [28]. These materials are generally doped with protonic acids such as aqueous hydrochloric acid $(\mathrm{HCl})$ to provide conductivity in the order of $1 \mathrm{~S} / \mathrm{cm}[20]$.

PANI is composed of aniline repeat units connected to form a backbone of alternating nitrogen atoms and benzene rings; it exists in a variety of forms that differ in chemical and physical properties [29]. It also comes in three classifications depending upon the degree of oxidation of the nitrogen atoms; the leucoemeraldine base (LB; fully oxidized form); the emeraldine base (EB; half oxidized form) and the pernigraniline base (PNB; fully oxidized form) [30,31].

PANI can be doped by protonation. The unusual properties of PANI arise because it is A-B nature, since most other conducting polymers are A-A type [15]. Of the three different states of oxidation, the one that can be doped to the highly conducting state is called "polyemeraldine". It consists of amine (-NH-) and imine (=N-) sites in equal proportions (Fig. 1). The imine sites are protonated to generate the bipolaron form (Fig. 2) [27].

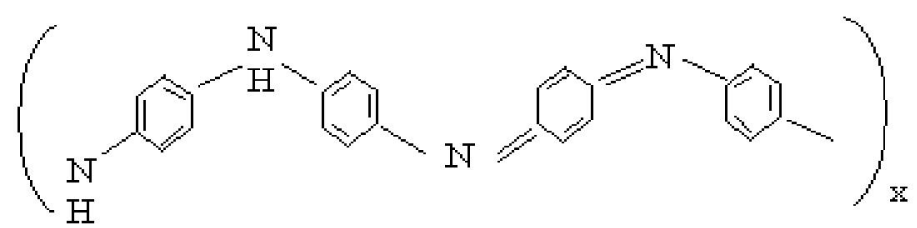

Figure 1: Emeraldine base basic (nonconducting) form of PANI -type polymer. 


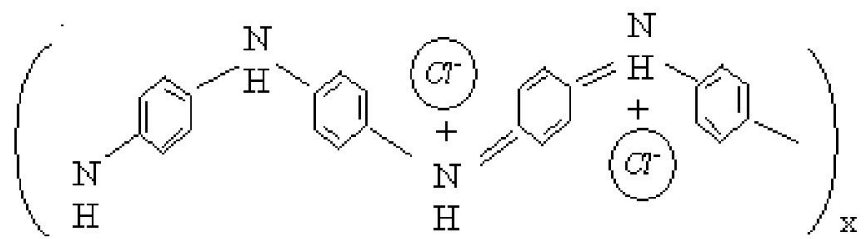

Figure 2: Emeraldine salt (conducting form) after protonation with $\mathrm{HCl}$

The EB can be transformed into a conducting state upon doping with protonic acids, such as $\mathrm{HCl}$ to yield the emeraldine salt (ES) $[20,27]$. It has been shown that protonation of PANI in the emeraldine oxidation state leads to a dramatic change in conductivity, from approximately $10^{10} \mathrm{~S} / \mathrm{cm}$ for the unprotonated polymer to a maximum conductivity of $10^{1}-10^{2} \mathrm{~S} / \mathrm{cm}$ when doped with aqueous acids [32,33]. The EB PANI has electrons spread throughout after doping [34, 31].

The present work reports the development of a conductive photoresist. The process of the development of films from the combination of EB PANI, SU-8 and NMP (N-methyl-2pyrrolidinone) is presented. These thin-films are studied in terms of their conductivity and morphology.

\section{EXPERIMENTS}

EB PANI (Sigma Aldrich) with a $\mathrm{Mw}$ of 214.27 was vacuum dried [35] for 12 hours in a to remove any moisture. 1-Methyl-2-pyrrolidone (NMP) of $99 \%$ purity from Sigma Aldrich was used as the solvent for the vacuum dried EB powder because NMP has non- chlorinated or chlorinated hydrocarbons [36] and is also commercially used as a SU-8 remover. It was reported by Kang et al. [37] that any polymer which is soluble in NMP can be blended with the NMP solution of EB.

The chemical polymerisation is carried out by dissolving various molecular weights of EB in NMP [5,20,34,38,28,31]. Initial tests for conductivity, were carried out with very low concentrations of EB $(\sim 0: 01 \mathrm{~g})$ in $5 \mathrm{ml}$ of NMP (mixture 1). Three different SU-8 viscosities, SU-8 2, SU-8 25 and SU-8 50 from Microchem were considered for experiments. The resultant mixture was passed through a syringe filter (pore size $0.2 \mathrm{~m}$ ). Concentrations from $0.05 \mathrm{~g} / \mathrm{ml}$ to $0.08 \mathrm{~g} / \mathrm{ml}$ of SU82 in "mixture 1" were spin coated on glass substrates at $3000 \mathrm{rpm}$ for $40 \mathrm{sec}$.

After curing on a hotplate for 5 minutes at $55^{\circ} \mathrm{C}$, the samples were soaked in $1 \mathrm{M}$ hydrochloric acid $(\mathrm{HCl})(33 \%$ purity) aqueous solution for 2 hours at room temperature. Because of the insulating nature of EB PANI, this soaking process protonates the polymer film. Therefore, the obtained chemical composition consists of emeraldine hydrochloride [20]. The resistance of the samples was measured using an ohm-meter (Fig. 3). The measured resistance was within the $\mathrm{k}$ to $M$ range. After protonation, the samples changed colour from dark blue to green. It was found that the greener the sample, the higher the conductivity. Thin films were sufficiently transparent for photolithographic applications.

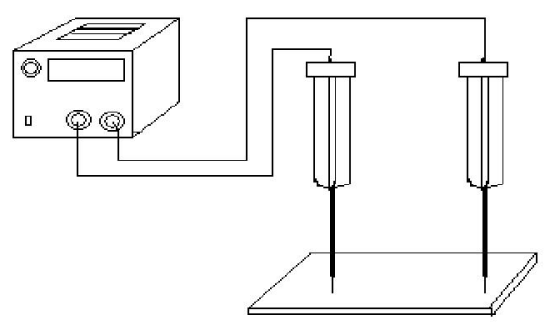

Figure 3: Resistance Measurement

It was concluded from the initial experiments that the resistance is measurable but the thickness of the polymer thin film deposited over the substrate was uneven because of low viscosity of the mixture. It was decided to increase the viscosity by using a higher concentration of SU-8 and EB.

"Mixture 2" was prepared by mixing $0.4 \mathrm{~g}$ of EB in $5 \mathrm{ml}$ of NMP. Greater percentage of SU-8 25 was added to increase the viscosity of samples. 10 samples with the volume in the range of $0.1 \mathrm{ml}$ to $1.0 \mathrm{ml}$ of SU-8 were mixed with $1.0 \mathrm{ml}$ of "mixture 2" in an ultrasonic bath for 72 hours. After spin coating and curing the samples, they were soaked in $1 \mathrm{M} \mathrm{HCl}$ (37\% purity) solution for 2 hours. The samples were air dried and measured for resistance using four-point probe technique as shown in Fig. $4[39,40]$.

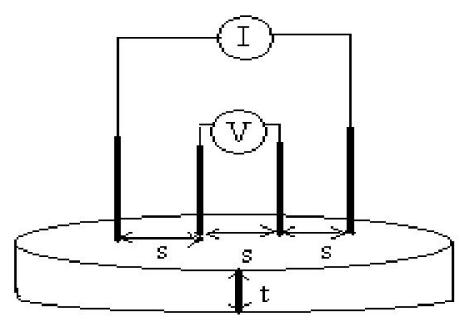

Figure 4: Four point resistance measurement technique

The four probe technique is a very efficient method for measuring the sheet resistance. It measures the sample resistance by measuring the current that flows for a given applied voltage. The outer two pins force a current through the sample and the inner two pins measure the voltage drop. In the case when the_ $1 \mathrm{~m}$ thickness is much smaller than the 
thickness of the substrate, the resistivity can be calculated using [39]:

$$
\rho=\frac{\pi}{\ln (2)} \frac{V}{I} \Omega c m
$$

(1)

Where, $\mathrm{t}$ is the thickness of the thin film, $\mathrm{V}$ is the measured voltage and $\mathrm{I}$ is the forced current.

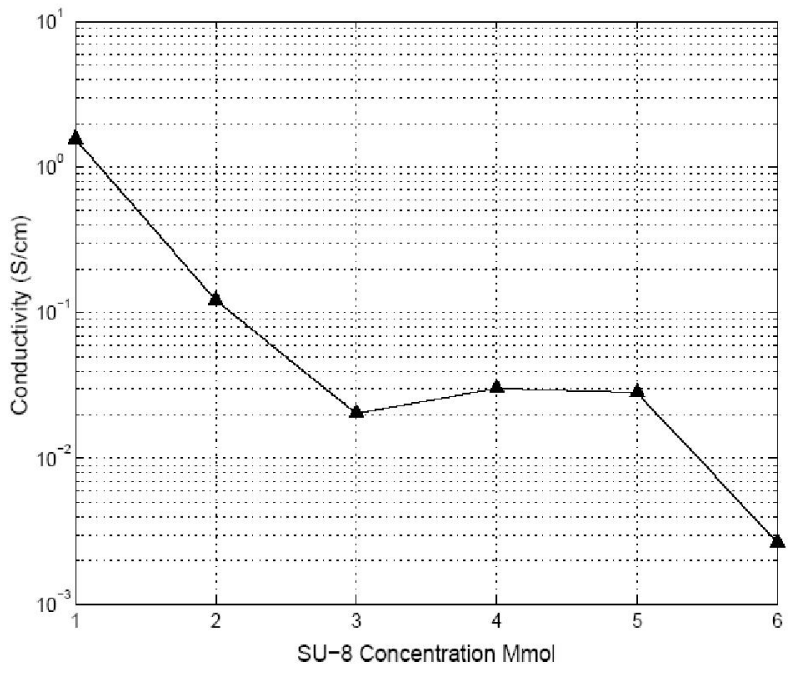

Figure 5: Average conductivity versus SU-8 volume percentage

Fig. 5 shows the average conductivity in all 10 samples of "mixture 2". The conductivity range was between $1.6 \mathrm{~S} / \mathrm{cm}$ and $2.5 \times 10^{-3} \mathrm{~S} / \mathrm{cm}$ for volume percentage of SU-8 in the range of 0.1 to 0.6 , respectively (Fig. 5).

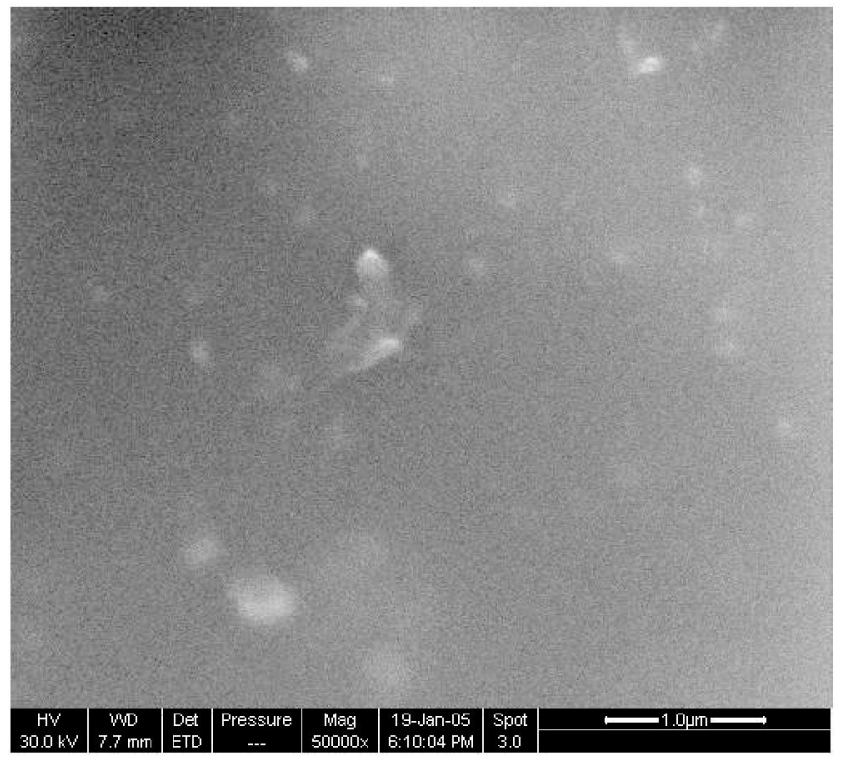

Figure 6: SEM Micro-graph of conductive thin film (0.4Mmol SU-8 and 1Mmol of "mixture 2")

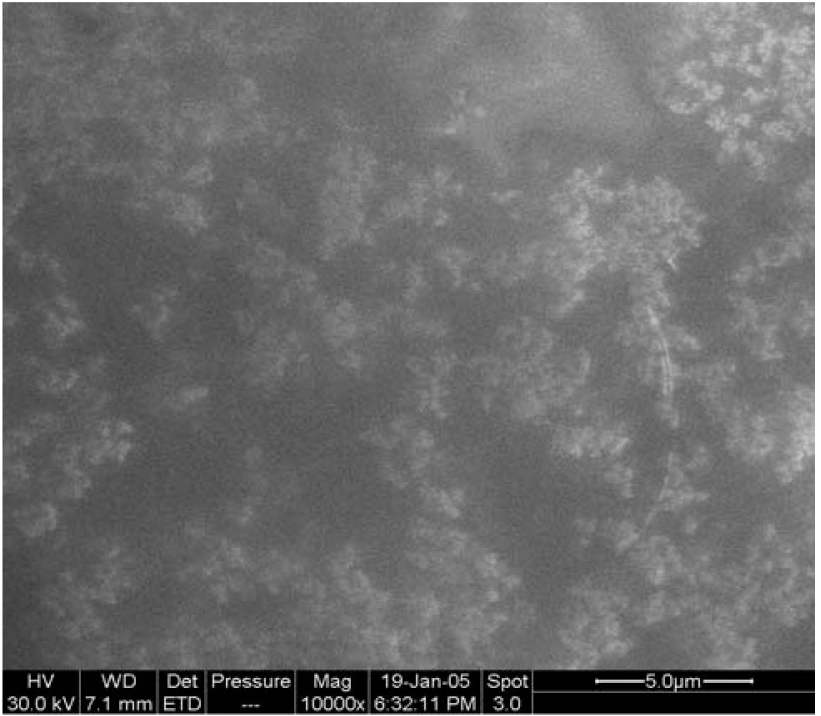

Figure 7: SEM Micro-graph of non-conductive thin film (0.9Mmol SU-8 and $1 \mathrm{Mmol}$ of "mixture 2")

Figures 6 and 7 depict SEM micro-graphs of the PANI/SU8 thin films. Fig. 6, which shows low concentration of SU-8 (0.4 Mmol SU-8 and 1Mmol of "mixture 2"), has particle dimensions ranging from approximately $50 \mathrm{~nm}$ to $200 \mathrm{~nm}$. It is observed that these particles are embedded in a continuous polymeric media. The film conductivity is approximately 0.03 $\mathrm{S} / \mathrm{cm}$.

Fig. 7, which shows high concentration of SU-8 (0.9 Mmol SU-8 and 1Mmol of "mixture 2"), has particle dimensions ranging approximately from $100 \mathrm{~nm}$ to $400 \mathrm{~nm}$. The materials are isolated clusters of particles with different dimensions.

These clusters are also located in a polymeric media. The thin film appears to be highly resistive, above the operating range of the measurement apparatus. It is suggested the formation of separate particles in this case reduces the continuity of PANI chains which reduces the conductivity.

\section{CONCLUSIONS AND FUTURE WORK}

A process for preparing chemically polymerized EB PANI with photoresist polymer SU-8 25 was developed. It was seen that for $0.1 \mathrm{Mmol}$ SU-8 in 1 Mmol of the mixture of EB PANI, and NMP, a conductivity as high as $1.6 \mathrm{~S} / \mathrm{cm}$ can be obtained. It was observed that increasing the weight percentage of $\mathrm{EB}$ in the mixture increases the conductivity. Morphology of the conductive and non-conductive thin films have been studied. In non- conductive thin films the media consists of separate clusters whilst the conductive thin films consists of nanoparticles embedded in a polymeric media. Properties of the thin films such as adhesion, spread viscosity 
and transparency of the material make the material suitable for applications such as biosensors. The novel photoresist could play an important role in developing structures such as conductive channels, gas sensors, biosensors and many more applications in the field of semiconductor device fabrication. Such a novel mixture can also be used for the development of high aspect ratio structures such as micro-needle biosensors which are generally developed using silicon material with a lengthy fabrication process.

In future work, it is intended to study the photolithographic properties of these thin films. The developed technique will be applied to produce thin layers of conductive photoresist for fabrication of various devices. It is also planned to develop high aspect ratio semiconductor structures.

\section{ACKNOWLEDGMENT}

This research is supported by a RIF grant from RMIT University.

\section{REFERENCES}

[1] Andreas Hierlemann, Oliver Brand, Christoph Hagleitner and Henry Baltes, "Microfabrication Techniques for Chemical/Biosensors", Proc IEEE, vol. 91, no. 6, June 2003, pp. 839-863.

[2] S. M. Sze, "Semiconductor Devices: Physics and Technology", 2nd ed, New York, Wiley, 2002.

[3] S. M Sze, Ed., "VLSI Technology", 2nd Ed, New York: McGraw Hill, 1988.

[4] C. Y. Chang, S. M Sze, Eds., "VLSI Technology", New York: McGraw Hill, 1996.

[5] M. Angelopoulos, "Conducting polymers in microelectronics", IBM J Res \& Dev, vol. 45, no. 1, January 2001, pp 67.

[6] S. Jiguer, A. Bertsch, H. Hofmann, P. Renaud, "Conductive SU8-silver composite photopolymer", Micromech. Sys. 17th IEEE Conf. on MEMS, 125-128, Jan 2004, pp. 125.

[7] N. LaBianca, J. D. Gelorme, "High aspect ratio for thick film applications", SPIE, vol. 2438, (1995), pp. 846-849.

[8] Eyre, J. Blosiu, D. Wiberg, "Taguchi optimization for the processing of EPON SU-8 resist", IEEE, 1998, pp. 218

[9] R. Ruchmann, K. Pfeiffer, M. Falenski, F. Reuther, R. Engelke and G. Grutzner, "SU-8 - a high performance material for MEMS applications", Polymers in MEMS, mstnews, pp. 45-46, http://www.microchem.com/resources/tok ebeam resist.pdf.

[10] J. Zhang, K. L. Tan, H. Q. Gong, "Characterization of the polymerization of SU-8 photoresist and its applications in micro-electro-mechanical systems (MEMS)", Polymer Testing, vol. 20, 2001, 693-701.

[11] M. Shaw, D. Nawrocki, R. Hurditch and D. Johnson, "Improving the process capability of SU-8", Microsystems Technologies 10, 2003, pp. 1-6.

[12] Y. J. Chuang, F. G. Tseng, W. K. Lin, "Reduction of diffraction effect of UV exposure on SU-8 negative thick photoresist by air gap elimination", Micorsys. Tech, vol. 8, 2002, pp. 308-313.

[13] Fred Williamson, Eric A. Shields, "SU-8 as an electron beam lithography resist", IEEE, 2003, pp. 57-60.

[14] J. M. Shaw, J. D. Galore, N. C. La Bionic, W. E. Coney, S. J. Holmes, "Negative photoresists for optical lithography", IBM Journal of Research \& Development, vol. 41 no. 1997, pp. 81-94.

[15] Peter G. Madden, John D. Madden, Patrick A. Anquetil, Hsiao-hua Yu, Timothy M. Swager and Ian W. Hunter, "Conducting polymers as building blocks for biomimetic systems", Bio-Robotics Symposium, The University of New Hampshire, USA, August 27 - 29, 2001.

[16] http://www.panipol.com/index.php Achieved on $27^{\text {th }}$ Jan '2005.

[17] Marco-A, De Paoli and Wilson A. Gazotti, "Electrochemistry, polymers and opto-electronic devices: A combination with a future", J. Braz Chem Soc, vol. 13, no. 4,2002 , pp. 410-424.
[18] Skotheim, T.A. Dekker, "Handbook of Conducting Polymers", New York, 1986.

[19] N. S. Sariciftci, A. J. Heeger, Y. Cao, "Paramagnetic susceptibility of highly conducting polyaniline: Disordered metal with weak electronelectron interactions (Fermi glass)", The American Phys. Soc. vol. 49, March 1994, pp. 5988-5992.

[20] A. G. MacDiarmid, J. C. Chiang, A. F. Richter, A. J. Epstein, "Polyaniline: A new concept in conducting polymers", Synthetic Metals, vol. 18, 1987, pp. .285-290.

[21] A. G. Green, A. E. Woodhead, Journal of Chemical Society, 2388, 1910.

[22] R. de Surville, M. Josefowics, L. T. Yu, J. Perichon and R. Buvet, "Electrochemical chains using protolytic organic semiconductors", Electrochem Acta, vol. 13, 1968, pp. 1451-1458.

[23] J. C. Chiang and A. G. MacDiarmid, "'Polyaniline': protonic acid doping of the emeraldine form to the metallic regime", Synthetic Metal, vol. 13, 1986, 193.

[24] P. Ghosh, A. Sarkar, M. Ghosh, A. K. Meikap, S. K. Chatoopadhyay, S K. Chatterjee, P. Chowdhury, B. Saha, "A study on hall voltage and electrical resistivity of doped conducting polyaniline", Czech. J. Phys., vol. 53, no. 12, 2003, pp. 1219-1227.

[25] Bhavana A. Deore, Insun Yu, Michael S. Freund, "A switchable self-doped polyaniline: interconversion between self-doped and non-self-doped forms", J. Am. Chem. Soc, vol. 126, 2004, pp. 52-53.

[26] Y. Cao, A. Andretta, A. J. Hegger and P. Smith, Polymer, vol. 30, 2305, 1989.

[27] Maja V, Majda Z, Marko Zupan, Anton S, "Influence of polymerization parameters on the molecular weight of polyaniline", Acta Chem. Slov vol. 45 , no. 2,1998 , pp. 173-183.

[28] Nureddin Colak, Bahar Sokmen, "Doping of chemically synthesized polyaniline", Designed monomers and polymers, vol. 3, no. 2, 181-189, 2000.

[29] Gordon G. Wallace, Georey M. Spinks, Peter R. Teasdale ConductiveElectroactive Polymers," Intelli. Mater. Sys. Technomic Publishing Company Inc., Lancaster, Basel, 1997.

[30] O. Kwon and M. L. McKee, "Calculation of Band Gaps in Polyaniline from Theoretical Studies of Oligomers" J. Phys. Chem B, vol. 104, 2000, pp. 1686-1694.

[31] M. Campos, Braz Bello Jr, "Mechanism of conduction in doped polyaniline", J. Phys. D: Appl. Phys., vol. 30, 1997, pp. 1531-1535.

[32] O. N. Timofeeva, B. Z. Lubentsov, Ye Z. Sudakova, D. N. Chernyshov, M. L. Khidekel, "Conducting polymer interaction with gaseous substances", I. water, Synth. Met, vol. 40, 1991, pp. 111-116.

[33] S. L. Hung, T. C. Wen, A. Gopalan, "Application of statistical design strategies to optimize the conductivity of electrosynthesized polypyrole", Mater. Lett, vol. 55, 2002, pp. 165-170.

[34] Kwang Sun Ryu, Soon Ho Chang, Seong-Gu Kang, Eung Ju Oh, Chul Hyun Yo, "Physicochemical and electrical characterization of polyaniline induced by crosslinking, stretching and doping", Bull. Koreann. Chem. Soc., vol. 20, no. 3, 1999, pp. 333-336.

[35] Dali Yang, Benjamin R Mattes, "Investigation of gel inhibitor assisted dissolution of polyaniline: a case study for EB, 2-methyl-aziridine and $\mathrm{n}$ methyl-pyrrolidine", Synthetic Metals, vol. 101, 1999, pp. 746-749.

[36] www.microchem.com/products/pdf/removerpg.pdf

[37] Yongku Kang, Sang Kim, Changjin Lee, "Doping of polyaniline by thermal acid-base exchange reaction", Materials Science and Engineering, vol. C 24, 2004, pp. 39-41.

[38] A. J. Epstein, J. M. Ginder, F. Zuo, R. W. Bigelow, H. S. Woo, D. B. Tanner, A. F. Richter, W. S. Huang, A. G. Mac Diarmid, "Insulator to metal transition in polyaniline", Synth. Metals, vol. 18, 1987, pp. 303-309.

[39] J. D. Plummer, M. D. Deal, P. B. Griffin, "Silicon VLSI technology, Fundamentals, Practice and Modeling," Printice Hall Inc., 2000 USA.

[40] Qingli Hao, Valentin Kulikov, Vladimir M. Mirsky, "Investigation of contact and bulk resistance of conducting polymers by simultaneous twoand four-point technique", Sensors and actuators, vol. B 94, 2003, pp. 352-357. 